Homer CS, Davis GK, Brodie PM, Sheehan A, Barclay LM, Wills J, Chapman MG. Collaboration in maternity care: a randomised controlled trial comparing community-based continuity of care with standard hospital care. British Journal of Obstetrics and Gynaecology 2001, 108, 16-22.

\title{
Collaboration in maternity care: a randomised controlled trial comparing community-based continuity of care with standard hospital care
}

Caroline SE Homer RM MN Senior Research Midwife

Gregory K Davis MD FRANZCOG Staff Specialist Obstetrician

Pat M Brodie RM MN Senior Research Midwife

Athena Sheehan RN MN Research Midwife

Lesley M Barclay RM PhD Professor of Family Health

Jo Wills RM MN Nursing and Patient Services Manager

Michael G Chapman MD FRANZCOG Professor of Obstetrics and Gynaecology.

Midwifery Practice and Research Centre

Centre for Family Health and Midwifery, South East Health, Faculty of Nursing, Midwifery and Health at the University of Technology Sydney and the Division of Women's and Children's Health, St George Hospital, Kogarah, New South Wales, 2217, Australia

Correspondence to CSE Homer: email homerc@sesahs.nsw.gov.au

\section{Sources of support:}

This study was partially funded by the Australian National Health and Medical Research Council and the New South Wales Health Department. 


\section{Abstract}

\section{Objective}

To test whether a new community-based model of continuity of care provided by midwives and obstetricians (the St George Outreach Maternity Project or STOMP) improved maternal clinical outcomes, in particular a reduced caesarean section rate.

\section{Design}

Randomised controlled trial.

\section{Setting}

A public teaching hospital in metropolitan Sydney.

\section{Sample}

1089 women randomised to either the STOMP model $(n=550)$ or standard hospital-based care $(n=539)$ prior to their first antenatal booking visit at an Australian metropolitan public hospital.

\section{Main outcome measures}

Data were collected on onset and outcomes of labour, antenatal, intrapartum and postnatal complications, antenatal admissions to hospital and neonatal mortality and morbidity.

\section{Results}

There was a significant difference in the caesarean section rate between the groups, $13.3 \%(73 / 550)$ in the STOMP group and $17.8 \%$ in the control group (96/539). This difference was maintained after controlling for known contributing factors to caesarean section $(\mathrm{OR}=0.6,95 \% \mathrm{Cl} 0.4-0.9, \mathrm{P}=0.02)$. There were no other significant differences in the events during labour and birth.

Eighty (14.5\%) neonates from the STOMP group and 102 (18.9\%) from the control group were admitted to the special care nursery but this difference was not significant (OR 0.75 , 95\% $\mathrm{Cl}$ 0.5-1.1, $\mathrm{P}=0.12$ ). Eight infants died during the perinatal period (four from each group), for an overall perinatal mortality rate of 7.3 per 1,000 births. 


\section{Conclusion}

Community-based continuity of maternity care provided by midwives and obstetricians resulted in a significantly reduced caesarean section rate. There were no other differences in clinical outcomes. 


\section{Introduction}

Australian public sector maternity services are mostly hospital-centred and provided in a fragmented manner. Women see a number of different health care providers (midwives, obstetricians, general practitioners) throughout their pregnancy and they are attended by different caregivers in labour and during the postnatal period. Numerous national and state government reports have recommended that services provide continuity of care and choice for women ${ }^{1-4}$ and reduce interventions in childbirth ${ }^{5}$.

Continuity of midwifery care has been shown to reduce interventions in labour, particularly augmentation of labour, analgesic use and electronic fetal monitoring. ${ }^{6-9} \mathrm{~A}$ small Canadian trial in 200 women demonstrated a significant reduction in caesarean section rate ${ }^{10}$ and an Australian trial reported a trend towards a reduced elective caesarean section rate in high risk women ${ }^{9}$. A retrospective cohort study in California has also shown that supportive nurse-midwifery care in labour was associated with a reduced caesarean section rate. ${ }^{11}$

This study was conducted in the context of both public and professional concern around the current system of midwifery care and as an attempt to implement a model of care that would result in improved clinical outcomes, particularly reduced caesarean section rates. We compared a community-based model of continuity of care involving both midwives and obstetricians, known as the St George Outreach Maternity Project (STOMP), with the standard model of hospital-based care.

\section{Methods}

\section{Study population}

The trial was conducted in a New South Wales public hospital situated in a metropolitan area. Thirty five percent of the population were born overseas with major cultural groups being from England, Greece, China, Italy, Egypt, Hungary and Lebanon. ${ }^{12}$ Women born in China or Hong Kong (4.8\%) and Lebanon (3.1\%) are the largest groups of non-English speaking childbearing women. ${ }^{13}$

Women were eligible for the trial if they were less than 24 weeks gestation at their first visit, lived in the designated catchment area and planned to have their baby in the delivery suite at the hospital. Exclusion criteria included the presence of significant maternal 
disease (for example, renal disease with impaired renal function, essential hypertension or insulin dependent diabetes), two previous caesarean sections or a previous classical caesarean section. Women who developed medical complications during their pregnancy remained in the group to which they were randomised.

\section{Intervention: the STOMP model of care}

Continuity of midwifery care was a focus of the STOMP model. A team of six full-time midwives provided care for 300 women per year that is, 50 births per midwife per year, or 25 births per month per team. Two teams were involved during the trial. The emphasis was on continuity of 'care' (a consistent team approach) rather than 'carer' (the same midwife).

Antenatal care was provided through two antenatal clinics in community centres. One clinic was based in an early childhood centre and the other in a family planning clinic. These sites were chosen due to the demographics of the areas, the suitability of the facilities and the accessibility and parking arrangements. Two midwives and an obstetrician or obstetric registrar attended each clinic. This meant that the STOMP team continued to care for women who developed complications antenatally and transfer to standard care was unnecessary. Women generally saw three or four of the STOMP midwives during the antenatal period. An informal 'meet the midwives' evening was held at each site every two months, so that women could have an opportunity to meet all six midwives if this was important to them.

One midwife from each STOMP team was always 'on call' for women in labour and to provide advice and information. This may have been a midwife that the woman had met in the antenatal period, but equally it may not have been. The 'on call' STOMP team midwife provided care during the labour and birth in the delivery suite at the hospital. For women who underwent an elective or an emergency caesarean section, the STOMP midwife continued to provide midwifery care in the operating theatre. If a woman required obstetric care during labour the obstetrician or registrar on call was contacted. This was not necessarily the doctor who had provided antenatal care.

After the birth, women could either choose to remain in hospital for postnatal care with STOMP midwives or be discharged early and receive domiciliary care by the STOMP midwives. 


\section{Control group: Standard care}

Standard care was provided in the hospital-based antenatal clinic, the delivery suite and the postnatal ward. Midwives and doctors saw women in the antenatal clinic. Women with risks were seen by an obstetrician or obstetric registrar. Low risk women were generally seen by midwives. Hospital-based antenatal care could also include visits to the women's general practitioner (GP) in a system known as GP shared care. Midwives and doctors on duty at the time provided care in the delivery suite and the postnatal ward.

Standard care was characterised by a lack of continuity of care across the antenatal, intrapartum and postnatal periods as a large number of clinicians provided care.

\section{Design}

The trial used the randomised consent design proposed by Zelen. ${ }^{18}$ Women were randomised to either the STOMP group or the control group (standard care) prior to obtaining consent. Women who were selected to the STOMP model were then offered this option. These women were still able to reject the offer and receive standard care, however they were still included in the analysis. Women in the control group were asked to participate in a satisfaction survey and received the standard hospital care. Records of women in the control group were not marked and their names were not available to the maternity staff.

The randomised consent method was chosen to overcome the potential bias that may exist when women become disappointed with their allocated group in the conventional consent-randomisation progression. Rowley et $\mathrm{al}^{9}$ reported that this disappointment may have contributed to the lower rates of satisfaction in their control group. This method of randomisation has also been used in two similar trials of new models of maternity care. ${ }^{6 ; 19}$ Prior to commencement, the trial was approved by the Institutional Ethics Committee of the South Eastern Sydney Area Health Service (Southern Section). Consent was sought from all the participants. Women in both groups were aware that they were part of a study. Control women were offered the standard care available at St George Hospital.

\section{Random allocation}

Assignment occurred prior to the woman's first hospital visit. Referral letters from general practitioners provided the information on which to register women in the trial. A preprepared list was generated using computer generated random numbers and women were 
stratified by parity. A remote randomisation system was used to ensure allocation concealment. The research midwife who was registering women in the trial telephoned an administrative assistant, who was not associated with the study in any other way, to receive each allocation. The allocation was not revealed until the woman's details were recorded on the list thus removing the chance of biasing the order in which women were registered.

\section{Outcome measures}

Outcome measures were chosen for their ability to reflect safety and efficacy in maternity care. The primary outcome measure was rate of caesarean section. Caesarean section rate was chosen as the primary outcome measure as it has important implications for women and health care services and appears to be reduced with continuity of care and supportive midwifery care in labour. ${ }^{9-11}$ This is an outcome that can be measured objectively. Secondary outcome measures included onset of labour, major complications, perineal trauma and neonatal admission to a special care nursery.

Data were collected from medical records by two experienced midwife researchers.

\section{Target sample size projection}

The sample size was calculated using the primary outcome measure, caesarean section rate. At significance level of 0.05 with $80 \%$ power, a sample of 1000 women was required to detect a fifty per cent reduction in the total caesarean section rate (from $10 \%$ to $5 \%$ ). The estimation of the current caesarean section rate reduction was based on previous research in our maternity unit demonstrating an emergency caesarean section rate of four per cent in low risk women attending the birth centre. ${ }^{14}$ Additional women were recruited to account for an anticipated 10 per cent attrition rate.

\section{Analysis}

Data were analysed using SPSS for Windows on an intention to treat basis. Categorical variables were analysed using chi-squared tests. Continuous variables with normal distributions were analysed using Student's t tests. Continuous data with a non-normal distribution were analysed using Mann Whitney $U$ tests. Logistic regression ${ }^{15}$ was used to control for factors that have previously been shown to affect the caesarean section rate (age, height, parity, history of a previous caesarean section, antenatal gestational diabetes, pre-eclampsia, antepartum haemorrhage and breech presentation at time of 
birth). Logistic regression was also used to look at factors associated with neonatal admission to the special care nursery. The $\alpha$ level for statistical significance was set at 0.05 .

The trial was too small to detect differences in perinatal mortality. An obstetrician, who did not work at the hospital and was unaware of the trial, the allocated groups or the ultimate aim of the review, 'blindly' assessed each perinatal death. The perinatal deaths were classified using the Maternal/Fetal Antecedents of Perinatal Mortality adapted from Whitfield et al. ${ }^{16}$ Perinatal mortality was defined as being a stillbirth (an infant of at least 20 weeks gestation or $400 \mathrm{gms}$ birth weight) or a neonatal death (the death of a live born infant within 28 days of life). ${ }^{17}$

\section{Results}

\section{Sample}

Between January 1997 and April 1998, 1282 women were randomised, 639 to the intervention group and 643 to the control group. As women were randomised four to five weeks before they were first seen in the antenatal clinic, a number cancelled their bookings. This was most commonly due to miscarriage or relocation to another hospital. There was no significant difference between the number of women lost from each group $(P=0.95)$. Women who had relocated to another hospital were removed as they had not attended the hospital for their first visit and were therefore unaware of their group allocation.

The final sample for analysis comprised of 1089 women, 550 in the STOMP group and 539 in the control group (Figure 1). Eighty-eight per cent (483/550) of women in the STOMP group received their allocated model of care. The reasons for refusal included: anxiety about giving information $(n=5)$; rather come to the hospital $(n=39)$; wanted birth centre care $(n=11)$ and not interested $(n=12)$. Ninety-four per cent of women in the control group agreed to participate in the satisfaction survey. Two women in the control group received STOMP care. This was an unintentional protocol violation that occurred when the women were erroneously offered the STOMP model. 


\section{Baseline characteristics}

Both groups were similar in demographic characteristics and past medical and obstetric history (Table 1). The proportions of the language groups were representative of the overall population of pregnant women attending the hospital. More than one fifth of women in the study required an interpreter, which was similar between the groups (21\% versus $22 \%)$.

\section{Antenatal care}

On average, women in the STOMP group attended 8.3 antenatal visits and women in the control group attended 7.4 antenatal visits. Sixteen per cent $(n=90)$ of women in the control group attended GP shared care, compared with 1.5 per cent $(n=8)$ in the STOMP group.

\section{Maternal outcomes}

Most women concluded their pregnancy at term with a mean gestation of 39 weeks (range 21-42) in both groups $(P=0.3)$.

There was a significant difference in the caesarean section rate between the groups, $13.3 \%(73 / 550)$ in the STOMP group and $17.8 \%$ (96/539) in the control group. This difference was maintained after controlling for the known contributing factors (Table 2). There were no other significant differences in events during labour and birth (Table 3).

More women from the control group were admitted to hospital during the antenatal period although this difference did not reach statistical significance. Attendance at the Day Assessment Unit for monitoring of hypertension and the frequency of antenatal complications were not significantly different between the groups (Table 4).

The mean postnatal length of hospital stay was slightly reduced in the STOMP group (4.9 days versus 5.1 days) but this difference was not significant. More women in the STOMP group utilised the early postnatal discharge program (43\% versus 35\%, OR $1.4,95 \% \mathrm{CI}$ 1.1-1.8, $P=0.003)$.

Twenty-one women (3.8\%) from the STOMP group and 14 (2.6\%) from the control group were readmitted at a mean of 3 weeks postpartum. The most common reasons were retained products of conception or endometritis (seven women from each group) and mastitis (five in the STOMP and three in the control group). 


\section{Neonatal outcomes}

In total 1099 neonates were born to the 1089 women in the study, with 10 sets of twins. There were no differences between the groups in mean birth weight $(3375 \mathrm{~g}$ in STOMP versus $3357 \mathrm{~g}$ in the control group, $\mathrm{P}=0.58$ ) or mean Apgar Scores (8.1 and 7.9 at one minute, $\mathrm{P}=0.2$ and 8.9 and 8.8 at five minutes, $\mathrm{P}=0.3)$. Twelve STOMP infants $(2.2 \%)$ and 13 control infants (2.4\%) had Apgar scores of less than 7 at five minutes $(p=0.8)$.

Eighty (14.5\%) neonates from the STOMP group and 102 (18.9\%) from the control group were admitted to the special care nursery but this difference was not significant (OR 0.75 , $95 \% \mathrm{Cl}$ 0.5-1.1, $\mathrm{P}=0.12$ ). The most important factors determining neonatal admission were gestation less than 37 weeks (OR 21.4, 95\% Cl 10.8-42.5, $\mathrm{P}<0.0001$ ), maternal antenatal risk factors (OR 3.5, 95\% Cl 2.3-5.3, $\mathrm{P}<0.001)$ and caesarean section (OR 1.6, 95\% Cl 1.03-2.5, $\mathrm{P}=0.03)$.

Eight infants died during the perinatal period (four from each group) with no deaths in the 10 twin pregnancies. Six of the infants were stillborn (four in the STOMP group and two in the control group) with two early neonatal deaths in the control group, giving a perinatal mortality rate of 7.3 per 1,000 births. A summary and review of the perinatal deaths is presented in Table 5. The only potentially preventable perinatal death was Case 1 (Table 5). This woman was a 32 year multipara with two previous uneventful pregnancies that resulted in normal vaginal births at term with normal birth weights. She received antenatal care at the STOMP clinic, attending regularly. Her fundal height at 28 weeks gestation was recorded at $27 \mathrm{~cm}$, at 31 weeks it was $31 \mathrm{~cm}$ and at 34 weeks it was $33 \mathrm{~cm}$, a total increase of six $\mathrm{cm}$ in six weeks. These last three recordings were by the same midwife. Fetal heart sounds were heard at all these visits and fetal movements reported by the woman. Her blood pressure was normal throughout the pregnancy. She presented at 36 weeks with no fetal movements for two days and a fundal height of $28 \mathrm{~cm}$. A fetal death in utero was diagnosed and induction of labour undertaken. A stillborn female (birth weight 1395gms) was born after a short labour. The autopsy reported an infant with moderate skin maceration and blistering and with weight and measurements equivalent to 31 weeks. The infant had no dysmorphic features but the placenta showed extensive infarction. All maternal pathology investigations were normal. 


\section{Discussion}

\section{Reduced caesarean section rate}

Our trial supports the hypothesis that continuity of maternity care provided in a collaborative manner by midwives and obstetricians in a community-based setting can result in a reduced caesarean section rate. Although these rates of caesarean section might seem high they are consistent with other Australian figures. ${ }^{20}$ The reduction in elective caesarean sections suggests the consistent relationship that the STOMP women developed with their midwives and obstetrician might have encouraged them to attempt a labour rather than elect surgery. The emergency caesarean reduction might be attributable to continuity of midwifery care during labour. Differences in caesarean section rates have not been demonstrated in most other trials of continuity of midwifery care,$^{21}$ although a trend to a reduction in high risk women has been reported. ${ }^{7 ; 9}$ A trial of 200 women in Canada did demonstrate a significant difference. ${ }^{10}$

The total caesarean section rate in the trial was higher than anticipated a priori. In 1997, the elective caesarean section rate at this hospital was $9.2 \%$ with a $10.3 \%$ emergency caesarean section rate. ${ }^{17}$ This suggests that the sample contained women with a range of obstetric risks and complications and was not exclusively 'low risk'. We believe this demonstrates that this model of care has utility in women with a range of pregnancyrelated risks and complications. Other interventions in labour, including analgesia, augmentation, electronic fetal monitoring and episiotomy were not altered. This possibly reflects the practice of the STOMP midwives, who were predominantly 'mainstream' midwives with similar experience and philosophies to those who provided standard care.

\section{Perinatal mortality}

While the study was not large enough to conclude whether the model of care was as safe as standard care in terms of perinatal mortality, a rate of 7.3 per 1000 births is comparable with other Australian data. In 1996, the perinatal mortality rate in Australia was 8.5 per 1000 births. ${ }^{20}$ In New South Wales, during the period 1993-97, the rate has ranged from 8.8 to 9.6 per 1000 births. ${ }^{17}$ Similar trials have shown a small, non-statistically significant increase in perinatal deaths associated with the continuity of care models ${ }^{6 ; 9 ; 19 ; 22 ; 23}$ and a recent systematic review cited this increase as a major concern. ${ }^{21}$ Our study is important as it adds to the relatively small numbers available for meta analysis to this point. 
The one death assessed to be potentially preventable was due to undiagnosed intrauterine growth restriction. However, it is accepted that the accuracy of measuring symphysiofundal height in predicting growth deficiency is poor. ${ }^{24}$

\section{Admission to SCN}

More infants in the control group were admitted to the special care nursery. This did not reach statistical significance, however the overall admission rates of 14.5 and 18.9 per cent in our study are of concern. There were no significant differences in birth weight or Apgar scores. The higher rates of neonatal admission in the control group may be related to the higher rate of caesarean section. Similar trials have reported mean admission rates of 6.1 per cent in continuity of care models and 8.9 per cent in standard care. ${ }^{21}$ This suggests that a low threshold for transfer exists, possibly enhanced by the close proximity of the delivery suite to the special care nursery and by protocols which encourage transfer. The admission rate may also reflect the range of obstetric risks in the sample, however, we believe the rate is still too high. Unnecessary separation of mother and baby has been reported to contribute to a delay in the establishment of breastfeeding ${ }^{25}$ and has cost implications for health services.

\section{Collaborative model of care}

This collaborative model of maternity care meant that women were not transferred to standard care if they developed pregnancy-related complications. Other models, which have catered for low risk women, have meant women who developed complication were transferred back to standard care when complications arose. In the Scottish trial, almost one third of women were permanently transferred from midwife-managed care, mainly for clinical reasons. ${ }^{26}$ In the Swedish trial of birth centre care, 13 per cent were transferred to standard care antenatally with a further 19 per cent transferring intrapartum ${ }^{22}$. Our collaborative approach also meant fewer women were admitted to hospital antenatally despite very similar rates of pregnancy-related complications.

\section{Community-based antenatal care}

The move towards community-based services is an ongoing goal of public health facilities. ${ }^{27}$ Despite this, community-based services offered as part of a planned continuity of care program encompassing labour and postnatal care, have not previously been reported or formally evaluated in Australia. Antenatal clinics in this trial were situated in a 
community-based setting to improve access and convenience for women, particularly those from disadvantaged groups and from non-English speaking backgrounds. The move to the community was not associated with any apparent detriment in the pregnancy outcomes of women.

Women in the STOMP group attended slightly more antenatal visits. This was because more women in the control group used GP shared care. This meant they had fewer antenatal visits recorded on their hospital records.

\section{Justification of the randomised consent design}

There has been concern expressed in the literature associated with trials that use the randomised consent method. ${ }^{28 ; 29}$ Our participants did not appear to be compromised in any way and were treated with respect. Consent was sought from all participants. Women in the STOMP group were able to refuse to participate. A small proportion (12\%) did refuse STOMP care, confirming our observation that women did not feel coerced or obliged to give consent. We believe that women were fully informed about their model of care.

\section{Limitations}

The trial was unblinded and it was not possible to mask the data collectors to the woman's allocation. Whilst this may be a limitation of the study, it is a common problem in research into health services. In an effort to reduce this bias the records of women in the control group were not identifiable to ensure that they received unaltered standard care. We also attempted to reduce bias by blinding the woman's allocated group from the reviewer of the eight perinatal deaths.

Twelve per cent of women in the STOMP group and 0.4 per cent of women from the control group did not receive the model of care to which they were randomly allocated. As the analysis was by intention to treat this may have impacted on the results.

\section{Conclusion}

The results of this trial suggest that community-based continuity of care provided collaboratively by midwives and obstetricians can result in a decreased caesarean section rate. Women who develop complications during pregnancy can continue to be managed in a community setting without apparent detriment to their health. The perinatal mortality rate was low although the study was insufficiently powered to assess true differences. 
The results from this trial can be aggregated with others in a similar manner to that conducted by Waldenström and Turnbull ${ }^{21}$ to further understand the clinical benefits associated with continuity of care in a public sector maternity service.

\section{Acknowledgments}

We would like to thank the midwives, obstetricians, obstetric registrars, interpreters, translators and clerical staff who were involved in the study and the staff at the Early Childhood Centre and the Family Planning Clinic who were generous in their support and encouragement. We are grateful for the statistical advice supplied by Dr Peter Petocz, for the ongoing statistical support from Dr Margaret Cooke and for useful comments on a draft from Dr Val Gebski. Finally, we would like to thank the women who participated.

\section{Conflict of interest}

None 


\section{References}

1. National Health and Medical Research Council (Aust). Options for effective care in childbirth. Canberra: Australian Government Printing Service; 1996.

2. Ministerial Task Force on Obstetric Services in NSW. Final Report of the Ministerial Task Force on Obstetric Services in NSW. Sydney: NSW Health Department; 1989.

3. Victorian Department of Health. Having a Baby in Victoria: Ministerial Review of Birthing Services in Victoria. Melbourne: Victorian Department of Health; 1990.

4. Michael CA. Ministerial Task Force to Review Obstetric, Gynaecological and Neonatal Services in Western Australia. Perth: Health Department of Western Australia; 1990.

5. Commonwealth Department of Health and Community Services. Goals and targets of Australia's health in the year 2000 and beyond. Canberra: AGPS; 1993.

6. Flint C, Poulengeris P, Grant A. The "Know Your Midwife' scheme - a randomised trial of continuity of care by a team of midwives. Midwifery 1989; 5: 11-16.

7. Hodnett ED. Continuity of care givers during pregnancy and childbirth [Cochrane Review] In: The Cochrane Library, Issue 3, Oxford: Update Software; 1998.

8. Waldenström $U$, Nilsson CA. A randomised controlled study of birth centre care versus standard maternity care: Effects on women's health. Birth 1997; 24:17-26.

9. Rowley MJ, Hensley MJ, Brinsmead MW, Wlodarczyk JH. Continuity of care by a midwife team versus routine care during pregnancy and birth: a randomised trial. Med J Aust 1995;163: 289-193.

10. Harvey S, Jarrell J, Brant R, Stainton C, Rach D. A randomised, controlled trial of nurse-midwifery care. Birth 1996;23:128-135.

11. Butler J, Abrams B, Parker J, Roberts JM, Laros RK. Supportive nurse-midwife care is associated with a reduced incidence of caesarean section. Am J Obstet Gynecol 1993; 168: 1407-1413. 
12. NSW Health Department. South Eastern Sydney Area Health Service Profile. Available from URL: http://internal.health.nsw.gov.au/areas/sesahs/profile.htm. 1999.

13. Nivison-Smith I. South Eastern Sydney Area Health Service Social and Demographic Characteristics. Summary Report. Sydney: South Eastern Sydney Multicultural Health Unit; 1998.

14. Homer CSE, Davis GK, Petocz P, Barclay LM, Matha D, Chapman MG. Birth centre or labour ward. A comparison of the clinical outcomes of low risk women in a NSW hospital. Aust J Adv Nurs 2000; 18: 8-12.

15. Hosmer DW, Lemeshow S. Applied Logistic Regression. New York: Wiley; 1989.

16. Whitfield CR, Smith NC, Cockburn F, Gibson AAM. Perinatally related wastage: A proposed classification of primary obstetric factors. Br J Obstet Gynaecol 1986; 93: 694-703.

17. NSW Health Department. New South Wales Mothers and Babies 1997. Sydney: NSW Health Department; 1998.

18. Zelen M. A new design for randomized controlled trials. N Engl J Med 1979; 33: 1242-1245.

19. MacVicar J, Dobbie G, Owen-Johnstone L, Jagger C, Hopkins M, Kennedy J: Simulated home delivery in hospital: a randomised controlled trial. $\mathrm{Br} \mathrm{J}$ Obstet Gynaecol 1993; 100:3 16-323.

20. Day P, Sullivan EA, Lancaster P. Australia's Mothers and Babies 1996. Sydney: Australian Institute of Health and Welfare National Perinatal Statistics Unit; 1999.

21. Waldenström $U$, Turnbull $D$. A systematic review comparing continuity of midwifery care with standard maternity services. Br J Obstet Gynaecol 1998; 105: 1160-1170.

22. Waldenström $U$, Nilsson $C A$, Winbladh B: The Stockholm birth centre trial. Maternal and infant outcome. Br J Obstet Gynaecol 1997; 104: 410-418.

23. Kenny P, Brodie P, Eckermann S, Hall J: Westmead Hospital Team Midwifery Project Evaluation: Final Report. Sydney: Westmead Hospital; 1994. 
24. Weiner CP: Fetal growth deficiency and its evaluation. In: James DK, Steer PJ, Weiner CP, Cronk B, editors. High Risk Pregnancy. London: WB Saunders; 1994. p. 760 .

25. Klaus M, Kennell J. Parent-infant bonding, St Louis: Mosby; 1982.

26. Turnbull D, Holmes A, Shields N, Cheyne H, Twaddle S, Gilmour WH, et al. Randomised, controlled trial of efficacy of midwife-managed care. Lancet 1996; 348: 213-218.

27. NSW Health Department. Strategic Directions for Health 1998-2003. Sydney: NSW Health Department; 1998.

28. McLean S. Commentary: No consent means not treating the patient with respect. BMJ 1997; 314: 1076-1077.

29. Smith R. Informed consent: the intricacies. BMJ 1997; 314: 1059-1060. 
Fig 1: Flow chart describing progress of participants

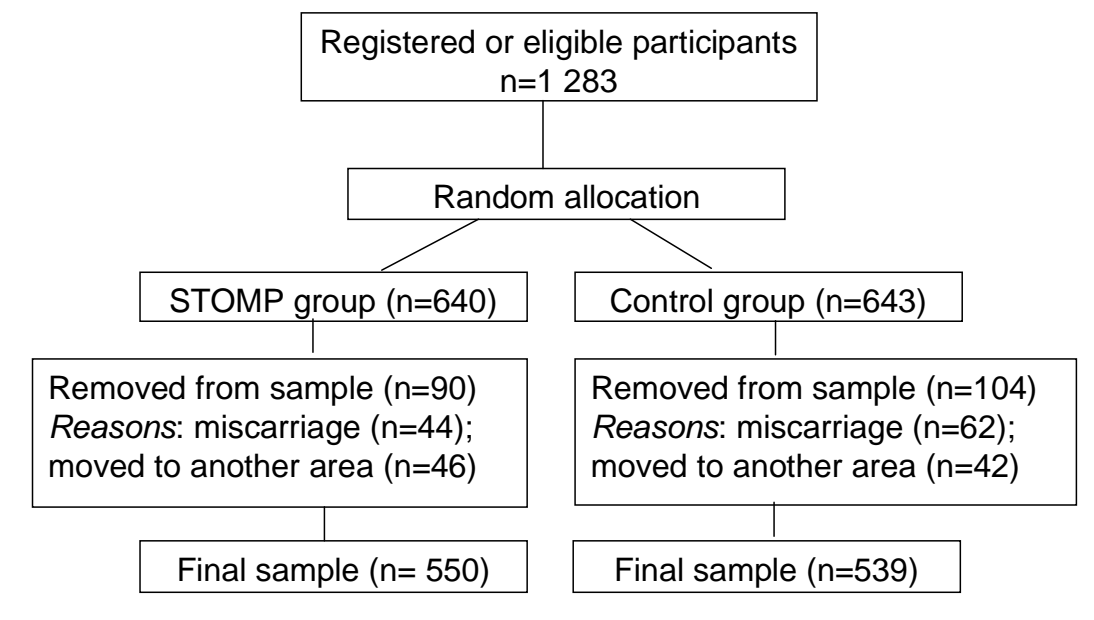


Table 1: Baseline maternal characteristics by allocated group

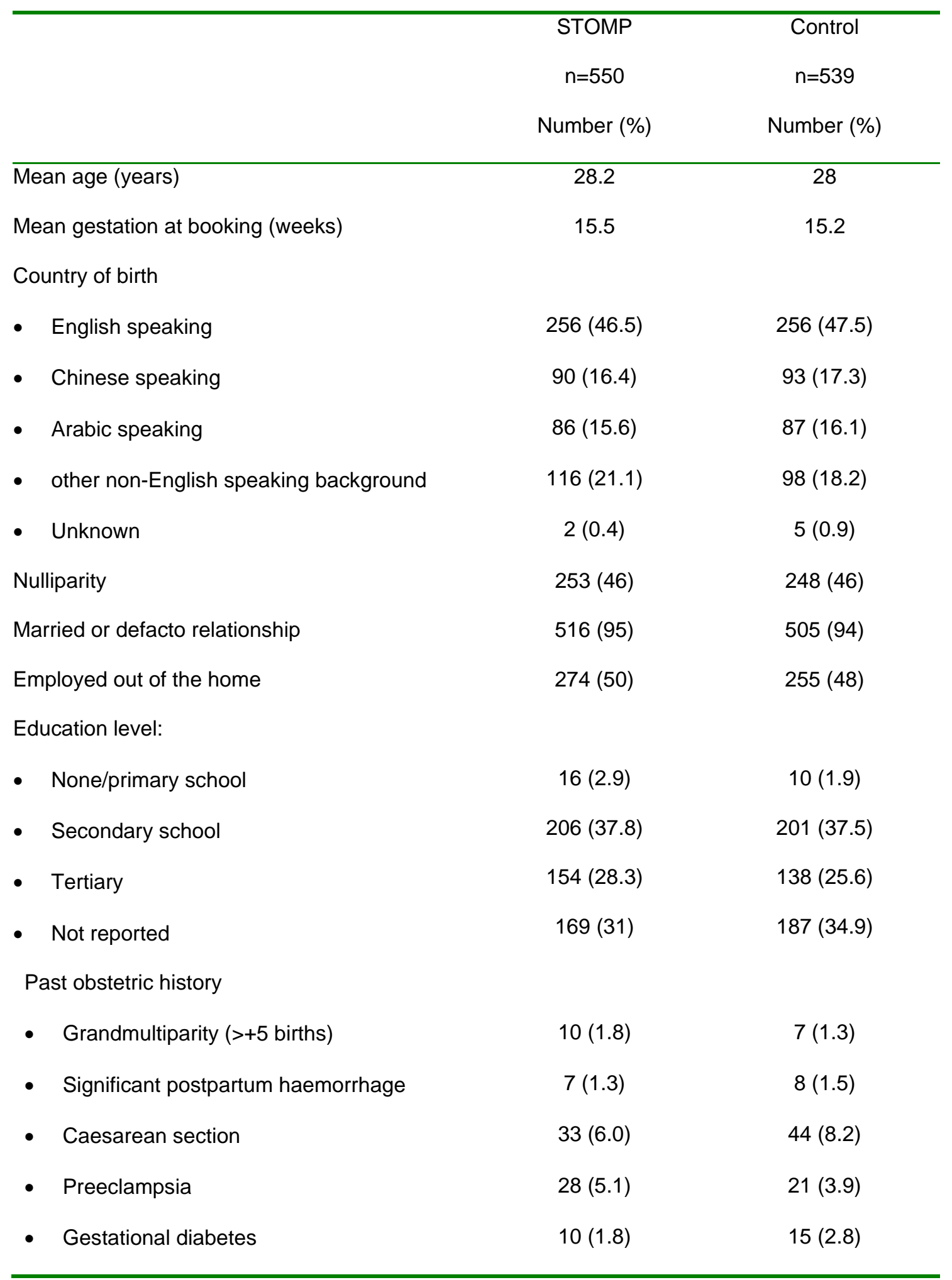


Table 2: Logistic regression with caesarean section as the dependent variable.

\begin{tabular}{lccc}
\hline Variable & $\mathrm{P}$ & OR & $95 \% \mathrm{Cl}$ \\
\hline Group (STOMP versus control) & 0.02 & 0.6 & $0.4-0.9$ \\
Parity & $<0.0001$ & 0.2 & $0.1-0.3$ \\
Obstetric risk factor & 0.008 & 1.9 & $1.2-3.0$ \\
Age & $<0.0001$ & 1.1 & $1.0-1.1$ \\
Height & 0.42 & 1.0 & $0.9-1.0$ \\
Previous caesarean section & $<0.0001$ & 33.9 & $17.2-67.0$ \\
\hline
\end{tabular}

Factors included in the model were allocated group (STOMP vs control), parity (nulliparity vs multiparity), age (continuous measure), height (continuous measure), obstetric risks (gestational diabetes, pre-eclampsia or antepartum haemorrhage vs none), previous caesarean section (previous caesarean vs no previous caesarean). 
Table 3: Events during labour and outcome of labour by allocated group

\begin{tabular}{lcc}
\hline & STOMP & Control \\
& $\mathrm{n}=550$ & $\mathrm{n}=539$ \\
& Number (\%) & Number (\%) \\
\hline Analgesia: & & \\
- Nitrous oxide & $360(65.4)$ & $325(60.3)$ \\
- Narcotic (pethidine) & $159(28.9)$ & $136(25.2)$ \\
- Epidural/spinal block & $157(28.5)$ & $172(31.9)$ \\
Induction of labour & $125(22.7)$ & $109(20.2)$ \\
Augmentation & $227(41.3)$ & $200(37.1)$ \\
Electronic fetal monitoring: & & \\
- during labour & $252(45.8)$ & $275(51)$ \\
Caesarean section: & $73(13.3)$ & $96(17.8)$ \\
- Elective & $21(3.8)$ & $34(6.3)$ \\
- Emergency & $52(9.5)$ & $62(11.5)$ \\
Forceps/Vacuum extraction & $71(12.9)$ & $63(11.7)$ \\
Episiotomy & $63(11.5)$ & $66(12.2)$ \\
Postpartum: & & $5(0.9)$ \\
\hline & & \\
\hline
\end{tabular}


Table 4: Antenatal complications by allocated group

\begin{tabular}{lcc}
\hline Complications & $\begin{array}{c}\text { STOMP group } \\
\mathrm{n}=550\end{array}$ & $\begin{array}{c}\text { Control group } \\
\mathrm{n}=539\end{array}$ \\
& Number (\%) & Number (\%) \\
\hline Antenatal admission & $53(9.6)$ & $72(13.4)$ \\
Day Assessment Unit attendance & $27(4.9)$ & $30(5.6)$ \\
Complications: & & \\
- Antepartum haemorrhage & $9(1.6)$ & $14(2.6)$ \\
- Preeclampsia & $33(6.0)$ & $34(6.3)$ \\
- Gestational diabetes & $42(7.6)$ & $37(6.9)$ \\
- Threatened preterm labour & $8(1.5)$ & $12(2.2)$ \\
- Other & $76(13.8)$ & $96(18)$ \\
\hline
\end{tabular}


Table 5: Perinatal mortality review of the eight perinatal deaths that occurred in the study.

\begin{tabular}{|c|c|c|c|c|c|}
\hline Case & Group & Gest & SB/NND & Timing & Reason for death $^{16}$ \\
\hline \multirow[t]{2}{*}{1} & STOMP & 36 & SB & Pre labour & Intrauterine growth restriction, Category 2.2 placental pathology. \\
\hline & & & & & Potentially preventable (probably small at 31-34 weeks but undetected) \\
\hline \multirow[t]{2}{*}{2} & STOMP & 31 & SB & Pre labour & Non-immune hydrops, Category 9.2 fetal abnormality cardiovascular system. \\
\hline & & & & & Unrelated to the model of care received \\
\hline \multirow[t]{2}{*}{3} & STOMP & 40 & SB & Pre labour & Unexplained, Category 3. \\
\hline & & & & & Unrelated to the model of care received \\
\hline \multirow[t]{2}{*}{4} & STOMP & 40 & SB & Intrapartum & Intrapartum asphyxia, Category 5.1. \\
\hline & & & & & Unrelated to the model of care received \\
\hline \multirow[t]{2}{*}{5} & Control & 39 & SB & Unknown & Unexplained, Category 3. \\
\hline & & & & & Unrelated to the model of care received \\
\hline \multirow[t]{2}{*}{6} & Control & 23 & NND & 12 hours & Preterm labour (?cervical incompetence), Category 14.5. \\
\hline & & & & & Unrelated to the model of care received \\
\hline \multirow[t]{2}{*}{7} & Control & 21 & SB & Unknown & Antepartum haemorrhage, Category 8.1 (abruption). \\
\hline & & & & & Unrelated to the model of care received \\
\hline \multirow[t]{2}{*}{8} & Control & 40 & NND & 12 hours & Acute chorioamnionitis and E coli pneumonia, Category 12.2. \\
\hline & & & & & Unrelated to the model of care received \\
\hline
\end{tabular}

NB: Case 4 was a woman who was offered STOMP care but chose to receive standard care through the antenatal clinic 
